\title{
ON GENERATING FUNCTIONS OF BIORTHOGONAL POLYNOMIALS SUGGESTED BY LAGUERRE POLYNOMIALS
}

\author{
B. C. Chandra ${ }^{1}$, K. P. Samanta ${ }^{2}$, C. S. Bera ${ }^{3}$ \\ ${ }^{1}$ Sree Vidyaniketan, 9A, J. M. Avenue, Kolkata - 700006 \\ ${ }^{2,3}$ Department of Mathematics, Bengal Engineering and Science University, Shibpur, \\ P.O. Botanic Garden, Howrah - 711 103, India \\ biplab_chandra72@yahoo.com, kalipadasamanta2010@gmail.com, chandrasekharbera75@gmail.com
}

\begin{abstract}
In this paper, we have obtained some novel generating functions (both bilateral and mixed trilateral) involving modified bi-orthogonal polynomials $Y_{n+m}^{\alpha+n}(x ; k)$, by group-theoretic method. As particular cases, we obtain the corresponding results on generalized Laguerre polynomials.
\end{abstract}

Key words: AMS-2000 Classification Code :33C45,33C47, Biorthogonal polynomials, Laguerre polynomials, generating functions.

\section{INTRODUCTION}

An explicit representation for the Konhauser bi-orthogonal polynomials [1], $Y_{n}^{\alpha}(x ; k)$, suggested by the Laguerre polynomials was given by carlitz [2] in the following form:

$$
Y_{n}^{\alpha}(x ; k)=\frac{1}{n !} \sum_{i=0}^{n} \frac{x^{i}}{i !} \sum_{j=0}^{i}(-1)^{j}\left(\begin{array}{l}
i \\
j
\end{array}\right)\left(\frac{j+\alpha+1}{k}\right)_{n},
$$

Where, $(a)_{n}$ is the pochhammer symbol, $\alpha>-1$ and $k$ is a positive integer.

The aim at presenting this paper is to obtain some results (new and known) on bilateral and mixed trilateral generating functions for the modified Konhauser biorthogonal polynomials $Y_{n+m}^{\alpha+n}(x ; k)$, by the group-theoretic method .Several particular cases of interest are also discussed in this paper. The main results of our investigation are stated in the form of the following theorems :

\section{Theorem 1:}

If there exists a unilateral generating relation of the form:

$$
G(x, w)=\sum_{n=0}^{\infty} a_{n} Y_{n+m}^{\alpha+n}(\mathrm{x} ; \mathrm{k}) w^{n},
$$

Then

$$
\begin{aligned}
& (1-k w)^{\frac{-(1+\alpha+m k)}{k}} \exp \left(x\left[1-(1-k w)^{\frac{-1}{k}}\right]\right) G(x(1 \\
& -k w), \quad t w(1-k w))
\end{aligned}
$$

$$
=\sum_{n=0}^{\infty} w^{n} \sigma_{n}(x, t)
$$

Where

$\sigma_{n}(x, t)=\sum_{p=0}^{n} a_{p} k^{n-p}\left(\begin{array}{c}n+m \\ n-p\end{array}\right) Y_{n+m}^{\alpha+p}(\mathrm{x} ; \mathrm{k}) t^{p}$.

\section{Theorem 2:}

If there exists a generating relation of the form:

$$
G(x, u, w)=\sum_{n=0}^{\infty} a_{n} Y_{n+m}^{\alpha+n}(\mathrm{x} ; \mathrm{k}) \mathrm{g}_{\mathrm{n}}(\mathrm{u}) w^{n}
$$

Where $\mathrm{g}_{\mathrm{n}}(\mathrm{u})$ is an arbitrary polynomial of degree $n$ and right hand series have formal power series expansion, then

$$
\begin{gathered}
(1-k w)^{\frac{-(1+\alpha+m k)}{k}} \exp \left(x\left[1-(1-k w)^{\frac{-1}{k}}\right]\right) G(x(1 \\
-k w), u, \quad v w(1-k w)) \\
=\sum_{n=0}^{\infty} w^{n} \sigma_{n}(x, u, v)
\end{gathered}
$$

Where

$$
\sigma_{n}(x, u, v)=\sum_{r=0}^{n} a_{r} k^{n-r}\left(\begin{array}{c}
n+m \\
n-r
\end{array}\right) Y_{n+m}^{\alpha+r}(\mathrm{x} ; \mathrm{k}) \mathrm{g}_{\mathrm{r}}(\mathrm{u}) v^{r} .
$$




\section{DERIVATION OF A NEW GENERATING}

\section{FUNCTION:}

At first we consider the following operator R:

$$
\begin{aligned}
R=x y^{-1} z \frac{\partial}{\partial x}+ & z \frac{\partial}{\partial y}+(k+1) z^{2} y^{-1} \frac{\partial}{\partial z} \\
+ & (1-x+k m) y^{-1} z
\end{aligned}
$$

Such that

$$
\begin{aligned}
& R\left(Y_{n+m}^{\alpha+n}(\mathrm{x} ; \mathrm{k}) y^{\alpha} z^{n}\right) \\
& =k(n+m+1) Y_{n+m+1}^{\alpha+n}(\mathrm{x} ; \mathrm{k}) y^{\alpha-1} z^{n+1} .
\end{aligned}
$$

The extended form of the group generated by $R$ is given as follows:

$$
\begin{aligned}
& e^{w R} f(x, y, z) \\
& \quad=\left(1-k w y^{-1} z\right)^{-\frac{(k m+1)}{k}} \exp (x \\
& \left.\quad-\frac{x}{\left(1-k w y^{-1} z\right)^{\frac{1}{k}}}\right)
\end{aligned}
$$

$\times f\left(\frac{x}{\left(1-k w y^{-1} z\right)^{\frac{1}{k}}}, \frac{y}{\left(1-k w y^{-1} z\right)^{\frac{1}{k}}}, \frac{z}{\left(1-k w y^{-1} z\right)^{1+\frac{1}{k}}}\right)$

Where $f(x, y, z)$ is an arbitrary function and $w$ is an arbitrary constant. Now using (2.3), we obtain

$e^{w R}\left(Y_{n+m}^{\alpha+n}(x ; k) y^{\alpha} z^{n}\right)=\left(1-k w y^{-1} z\right)^{-\frac{(k m+k n+\alpha+n+1)}{k} y^{\alpha} z^{n}}$

$$
\begin{gathered}
\times \exp (x \\
\left.-\frac{x}{\left(1-k w y^{-1} z\right)^{\frac{1}{k}}}\right) Y_{n+m}^{\alpha+n}\left(\frac{x}{\left(1-k w y^{-1} z\right)^{\frac{1}{k}}} ; k\right) .
\end{gathered}
$$

But using (2.2), we obtain

$e^{w R}\left(Y_{n+m}^{\alpha+n}(x ; k) y^{\alpha} z^{n}\right)=\sum_{p=0}^{\infty} \frac{w^{p}}{p !} R^{p}\left(Y_{n+m}^{\alpha+n}(x ; k) y^{\alpha} z^{n}\right)$

$=\sum_{p=0}^{\infty}\left(\begin{array}{c}n+m+p \\ n+m\end{array}\right) Y_{n+m+p}^{\alpha+n}(x ; k) y^{\alpha} z^{n}\left(k w y^{-1} z\right)^{p}$.
Equating (2.4) and (2.5) and then putting $k w y^{-1} z=w$, we get

$$
\begin{aligned}
(1-w)^{-\frac{(1+\alpha+n+n k+m k)}{k}} \exp (x \\
\left.-\frac{x}{(1-w)^{\frac{1}{k}}}\right) Y_{n+m}^{\alpha+n}\left(\frac{x}{(1-w)^{\frac{1}{k}}} ; k\right) \\
=\sum_{p=0}^{\infty}\left(\begin{array}{c}
n+m+p \\
n+m
\end{array}\right) Y_{n+m+p}^{\alpha+n}(x ; k) w^{p} .
\end{aligned}
$$

Now putting $m=0$ in (2.6), we get

$$
\begin{aligned}
&(1-w)^{-\frac{(1+\alpha+n+n k)}{k}} \exp (x \\
&\left.-\frac{x}{(1-w)^{\frac{1}{k}}}\right) Y_{n}^{\alpha+n}\left(\frac{x}{(1-w)^{\frac{1}{k}}} ; k\right) \\
&=\sum_{p=0}^{\infty}\left(\begin{array}{c}
n+p \\
n
\end{array}\right) Y_{n+p}^{\alpha+n}(x ; k) w^{p},
\end{aligned}
$$

Which does not seem to have appeared in the earlier works. Now putting $\mathrm{n}=0$ and replacing $\alpha$ by $\alpha-n$ in (2.6), we get

$$
\begin{aligned}
& (1-w)^{-\frac{(k m+\alpha+1)}{k}} \exp \left(x-\frac{x}{(1-w)^{\frac{1}{k}}}\right) Y_{m}^{\alpha}\left(\frac{x}{(1-w)^{\frac{1}{k}}} ; k\right) \\
& =\sum_{p=0}^{\infty}\left(\begin{array}{c}
m+p \\
m
\end{array}\right) Y_{m+p}^{\alpha}(x ; k) w^{p}
\end{aligned}
$$

Which is found derived in [3] by the classical method. Now putting $\mathrm{m}=0$ in (2.8), we get

$$
\begin{array}{r}
(1-w)^{-\frac{(1+\alpha)}{k}} \exp \left(x-\frac{x}{(1-w)^{\frac{1}{k}}}\right) \\
=\sum_{p=0}^{\infty} Y_{p}^{\alpha}(x ; k) w^{p},
\end{array}
$$

Which is found derived in $[3,4]$.

\section{Special Case 1:}

If we put $k=1$, then $Y_{n}^{\alpha}(x ; k)$ reduce to the generalized Laguerre polynomials, $L_{n}^{\alpha}(x)$. Thus Putting $k=1$ in the above generating relations, we get the following generating relation on Laguerre polynomials: 


$$
\begin{array}{r}
(1-w)^{-(1+\alpha+2 n)} \exp \left(\frac{-x w}{1-w}\right) L_{n}^{(\alpha+n)}\left(\frac{x}{1-w}\right) \\
=\sum_{p=0}^{\infty}\left(\begin{array}{c}
n+p \\
p
\end{array}\right) L_{n+p}^{(\alpha+p)}(x) w^{p} \\
(1-w)^{-(1+\alpha+m)} \exp \left(\frac{-x w}{1-w}\right) L_{m}^{(\alpha)}\left(\frac{x}{1-w}\right) \\
=\sum_{p=0}^{\infty}\left(\begin{array}{c}
m+p \\
p
\end{array}\right) L_{m+p}^{(\alpha)}(x) w^{p}
\end{array}
$$

And

$(1-w)^{-(1+\alpha)} \exp \left(\frac{-x w}{1-w}\right)=\sum_{p=0}^{\infty} L_{p}^{(\alpha)}(x) w^{p}$.

The generating functions (2.11) and (2.12) are found derived in $[5,6,7]$.

\section{PROOF OF THEOREM 1}

Let us consider the generating relation of the form:

$G(x, w)=\sum_{n=0}^{\infty} a_{n} Y_{n+m}^{\alpha+n}(x ; k) w^{n}$

Replacing $w$ by $t w z$ and multiplying both sides of (3.1) by $y^{\alpha}$ and finally operating $e^{w R}$ on both sides, we get

$e^{w R}\left(y^{\alpha} G(x, t w z)\right)$

$=e^{w R}\left(\sum_{n=0}^{\infty} a_{n}\left(Y_{n+m}^{\alpha+n}(x ; k) y^{\alpha} z^{n}\right)(t w)^{n}\right)$.

Now the left member of (3.2), with the help of (2.3), reduces to

$$
\begin{aligned}
& \left(1-k w y^{-1} z\right)^{-\frac{(\alpha+k m+1)}{k}} \exp \left(x-\frac{x}{\left(1-k w y^{-1} z\right)^{\frac{1}{k}}}\right) y^{\alpha} \\
\times & \left.G\left(\frac{x}{\left(1-k w y^{-1} z\right)^{\frac{1}{k}}}, \quad \frac{t w z}{\left(1-k w y^{-1} z\right)^{1+\frac{1}{k}}}\right) \cdot 3.3\right)
\end{aligned}
$$

The right member of (3.2), with the help of (2.2), becomes

$$
\begin{gathered}
\sum_{n=0}^{\infty} \sum_{p=0}^{n} a_{n} \frac{w^{p}}{p !} k^{p}(n+m \\
+1)_{p} Y_{n+m+p}^{\alpha+n}(x ; k) y^{\alpha-p} z^{n+p}(t w)^{n} \\
=\sum_{n=0}^{\infty} \sum_{p=0}^{n} a_{n-p} \frac{w^{n}}{p !} k^{p}(n-p+m \\
+1)_{p} Y_{n+m}^{\alpha+n-p}(x ; k) y^{\alpha-p} z^{n}(t)^{n-p}(3.4)
\end{gathered}
$$

Now equating (3.3) and (3.4) and then substituting $y=z=1$, we get

$$
\begin{aligned}
& (1-k w)^{-\frac{(1+m k+\alpha)}{k}} \exp (x \\
& \left.-\frac{x}{(1-k w)^{\frac{1}{k}}}\right) G\left(\frac{x}{(1-k w)^{\frac{1}{k}}}, \frac{w t}{(1-k w)^{1+\frac{1}{k}}}\right) \\
& =\sum_{n=0}^{\infty} \sum_{p=0}^{n} a_{n-p} \frac{w^{n}}{p !} k^{p}(n-p+m \\
& \quad+1)_{p} Y_{n+m}^{\alpha+n-p}(x ; k) t^{n-p} .
\end{aligned}
$$

Therefore

$$
\begin{aligned}
& (1-k w)^{-\frac{(1+m k+\alpha)}{k}} \exp (x \\
& \left.-\frac{x}{(1-k w)^{\frac{1}{k}}}\right) G\left(\frac{x}{(1-k w)^{\frac{1}{k}}}, \frac{w t}{(1-k w)^{1+\frac{1}{k}}}\right) \\
& =\sum_{n=0}^{\infty} w^{n} \sigma_{n}(x, t),
\end{aligned}
$$

Where

$\sigma_{n}(x, t)=\sum_{p=0}^{n} a_{p} k^{n-p}\left(\begin{array}{c}n+m \\ n-p\end{array}\right) Y_{n+m}^{\alpha+n-p}(x ; k) t^{p}$.

This completes the proof of the theorem which is believed to be the new one.

\section{PROOF OF THEOREM 2:}

Again Let us consider the another generating relation of the form:

$G(x, u, w)=\sum_{n=0}^{\infty} a_{n} Y_{n+m}^{\alpha+n}(x ; k) g_{n}(u) w^{n}$.

Replacing $w$ by $w v z$ and then multiplying both sides of (4.1) by $y^{\alpha}$ and finally applying the operator $e^{w R}$ on both sides, we easily obtain, as in section 3 , the following generating relation:

$$
\begin{gathered}
\left(1-k w y^{-1} z\right)^{-\frac{(1+m k+\alpha)}{k}} \exp (x \\
\left.-\frac{x}{\left(1-k w y^{-1} z\right)^{\frac{1}{k}}}\right) y^{\alpha}
\end{gathered}
$$




$$
\begin{aligned}
& \times G\left(\frac{x}{\left(1-k w y^{-1} z\right)^{\frac{1}{k}}}, u, \frac{w v z}{\left(1-k w y^{-1} z\right)^{1+\frac{1}{k}}}\right) \\
& =\sum_{n=0}^{\infty} \sum_{r=0}^{n} a_{n-r} \frac{w^{n}}{r !} k^{r}(n-r+m \\
& \quad+1)_{r} Y_{n+m}^{\alpha+n-r}(x ; k) g_{n-r}(u) y^{\alpha-r} z^{n} v^{n-r}
\end{aligned}
$$

Now substituting $y=z=1$, we get

$$
\begin{aligned}
&(1-k w)^{-\frac{(1+m k+\alpha)}{k}} \exp \left(x-\frac{x}{(1-k w)^{\frac{1}{k}}}\right) \\
& \times G\left(\frac{x}{(1-k w)^{\frac{1}{k}}}, u, \frac{w v}{(1-k w)^{1+\frac{1}{k}}}\right) \\
&=\sum_{n=0}^{\infty} w^{n} \sigma_{n}(x, u, v),
\end{aligned}
$$

Where

$\sigma_{n}(x, u, v)=\sum_{r=0}^{n} a_{r} k^{n-r}\left(\begin{array}{c}n+m \\ n-r\end{array}\right) Y_{n+m}^{\alpha+r}(x ; k) g_{r}(u) v^{r}$.

This completes the proof of the theorem which is believed to be the new one.

\section{Special Case 2:}

If we put $k=1$ in our Theorem $1 \& 2$, we get the following result on generalized Laguerre polynomials:

\section{Theorem 3:}

If there exists a generating relation of the form:

$G(x, w)=\sum_{n=0}^{\infty} a_{n} L_{n+m}^{(\alpha+n)}(\mathrm{x}) w^{n}$,

Then

$$
\begin{gathered}
(1-w)^{-(1+\alpha+m)} \exp \left(\frac{-w x}{1-w}\right) G\left(\frac{x}{1-w}, \frac{t w}{(1-w)^{2}}\right) \\
=\sum_{n=0}^{\infty} w^{n} \sigma_{n}(x, t)
\end{gathered}
$$

Where

$\sigma_{n}(x, t)=\sum_{p=0}^{n} a_{p}\left(\begin{array}{c}n+m \\ n-p\end{array}\right) L_{n+m}^{(\alpha+p)}(\mathrm{x}) t^{p}$,

Which does not seem to have appeared in the earlier works.

\section{Theorem 4:}

If there exists a generating relation of the form:

$G(x, u, w)=\sum_{n=0}^{\infty} a_{n} L_{n+m}^{(\alpha+n)}(\mathrm{x}) g_{n}(u) w^{n}$,

Then

$$
\begin{gathered}
(1-w)^{-(1+\alpha+m)} \exp \left(\frac{-w x}{1-w}\right) \times G\left(\frac{x}{1-w}, u, \frac{t w}{(1-w)^{2}}\right) \\
=\sum_{n=0}^{\infty} w^{n} \sigma_{n}(x, u, v),
\end{gathered}
$$

Where

$\sigma_{n}(x, u, v)=\sum_{r=0}^{n} a_{r}\left(\begin{array}{c}n+m \\ n-r\end{array}\right) L_{n+m}^{(\alpha+r)}(\mathrm{x}) g_{r}(u) v^{r}$,

Which does not seem to have appeared in the earlier works.

\section{REFERENCES}

[1] Konhauser, J.D.E., Biorthogonal polynomials suggested by the Laguerre polynomials, Pacific J.Math., 21(1967), 303-314. [2] Carlitz, L.,A note on certain biorthogonal polynomials, Pacific J.Math.,24(1968),425-430.

[3] Prabhakar, T.R., On the other set of biorthogonal polynomials suggested by the Laguerre polynomials, Pacific J.Math., 37(1971), 801-804.

[4] Srivastava, H.M., Some Biorthogonal polynomials suggested by the Laguerre polynomials, Pacific J.Math.,98(1982)235-250.

[5] E.D.Rainville, Special Functions, Macmillan, New York, 1960.

[6] Truesdell, C., An essay toward a unified theory of special functions, Princeton university press, 1948.

[7] McBride, E.B., Obtaining Generating Functions, Springer Verlag, Berlin, 1972. 